\title{
Acknowledgment to Reviewers of Journal of Composites Science in 2020
}

\author{
Journal of Composites Science Editorial Office
}

MDPI AG, St. Alban-Anlage 66, 4052 Basel, Switzerland

Citation: Journal of Composites Science Editorial Office. Acknowledgment to Reviewers of Journal of Composites Science in 2020. J. Compos. Sci. 2021, 5, 38. https://doi.org/10.3390/jcs5020038

Published: 25 January 2021

Publisher's Note: MDPI stays neutral with regard to jurisdictional claims in published maps and institutional affiliations.

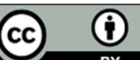

Copyright: () 2021 by the author. Licensee MDPI, Basel, Switzerland. This article is an open access article distributed under the terms and conditions of the Creative Commons Attribution (CC BY) license (http://creativecommons.org/licenses/by/4.0/).
Peer review is the driving force of journal development, and reviewers are gatekeepers who ensure that the Journal of Composites Science maintains high standards for the quality of its published papers. Thanks to the cooperation of our reviewers, in 2020, the median time taken for the first decision to be made was 12 days, and the median time to publication was 32 days. The editors would like to express their sincere gratitude to the following reviewers for their invaluable time and dedication, regardless of whether the papers were finally published:

Abdullah, Nur Azam
Abedin, Farhana
Ahmadi, Soha
Ahmed, Arif
Ahmed, Hawreen Hasan
Ahmed, Kumkum
Aida Alejandra, Pérez-Fonseca
Àkermo, Malin
Albiez, Matthias
Alhwaige, Almahdi
Allegra, Alessio
Almeida Jr, Humberto
Andrea, Ianni
Andrzejewski, Jacek
Anim-Danso, Emmanuel
Aniskevich, Andrey
Anyszka, Rafał
Arena, Maurizio
Arias, Manuel J. Lis
Arjmandi, Reza
Arteiro, Albertino
Artero Guerrero, Jose Alfonso
Asandulesa, Mihai
Ateia, Mohamed
Aymerich, Francesco
Babu, Libin K.
Badhe, Ravindra
Baird, Donald G.
Bajpai, Ankur
Banerjee, Shib Shankar
Bao, Weizhai
Bao, Yi
Barczewski, Mateusz
Alem

Barkoula, Nektaria-Marianthi

Barszczewska-Rybarek, Izabela

Baskonus, Haci Mehmet

Baumeister, Joachim

Bellisario, Denise

Beltsios, Konstantinos

Bhudolia, Somen K.

Bienias, Jaroslaw

Binetruy, Christophe

Birajdar, Mallinath S

Bonten, Christian

Borowicz, Marcin

Bortner, Michael

Bou, Santiago Ferrandiz

Bourmaud, Alain

Brožovský, Jiří

Bulgariu, Laura

Cai, Gaochuang

Calabrese, Luigi

Calafel, Itxaso

Camargo, Felipe Vannucchi De

Camposo Pereira, Artur

Cavallaro, Giuseppe

Caydamli, Yavuz

Cech, Vladimir

Cerruti, Pierfrancesco

Cesano, Federico

Chaitanya, Saurabh

Chang, Boon Peng

CHAVALI, Murthy

Cheng, Qingqing

Cheraghian, Goshtasp

Chevali, Venkata 
Cho, Jung Sang

Chowdhury, Reaz A

Chun, Sungwoo

Cohen, Yachin

Cortese, Marta

Cruz-Delgado, Víctor Javier

Cui, Wenyuan

Dan, Dobrota

Danlée, Yann

De Aguiar, José Barroso

De Camargo, Felipe Vannucchi

Del Barrio, Jesús

Dey, Vikram

Dhakal, Hom

Dhaliwal, Gurjot

Dhaliwal, Gurpinder Singh

Dimos, Konstantinos

Doitrand, Aurélien

Domínguez, María Elena Alemán

Dong, Chensong

Drissi-Habti, Monssef

Drobiec, Łukasz

Drozdov, A.D.

Dudás, Zoltán

Dufrasne, Isabelle

Dulska, Agnieszka

Dunky, Manfred

Durkin, David P.

Echtermeyer, Andreas

El-Dessouky, Hassan

Eleftheriadis, Georgios

Elhattab, Ahmed

Elsharafi, Mahmoud

Erikson, Heiki

Eslami, Hossein

Eum, Kiwon

Eyckens, Daniel J.

Fan, Jizhou

Fan, Zhaoyang

Fare, Silvia

Favaloro, Anthony

Ferdous, Wahid

Férec, Julien

Fernandes, Emanuel M.

FERNANDEZ- LOPEZ, Antonio

Ferreira, Filipe

Fidan, Ismail

Fiol, Nuria

Fitoussi, Joseph

Formela, Krzysztof

Franco, Camilo Andres
Franz, Gerald

$\mathrm{Fu}$, Feng

$\mathrm{Fu}$, Kun

Gabrion, Xavier

Ganguly, Sayan

Gao, Yuesheng

Garbiec, Dariusz

Garcés Osado, Andrés

Garcia Filho, Fabio Da Costa

Garoz, David

Garzon Hernandez, Sara

Gavaldà Diaz, Oriol

Gbaguidi, Audrey

Geier, Norbert

Ghazimoradi, Mehdi

Giannakas, Aris

Giner-Tarrida, Luís

Gniewosz, Małgorzata

Goh, Guo Dong

Gohari, Soheil

Gong, Hugh

Gordon, Wesley O.

Griggio, Flavio

Guillamet, Gerard

Gupta, Jaipal

Gurka, Martin

Haider, Julfikar

Hamama, Hamdi H. H.

Han, Yan

Hanif, Asad

Hanifehzadeh, Mohammad

Haponiuk, Józef

Hardisson, Arturo

Hasan, M. M. B.

Hassanin, Hany

Hatada, Ruriko

Hay, Ratana

Hayashi, Fumitaka

$\mathrm{He}$, Guanjie

Henry, Todd C.

Herbrand, Martin

Hornig, Andreas

$\mathrm{Hu}$, Anming

Hussain, Ghulam

Inam, Fawad

Irez, Alaeddin Burak

Ismael, Mohammed

Izadgoshasb, Iman

Jakić, Miće

James, Robin

James, Robinson 
Janovák, László

Jayachandran, K. P.

Jun, Byung-Moon

Jung, In Hwa

Kádár, Csilla

Kamarian, Saeed

Kamkar, Milad

Kandhasamy, Sathiyaraj

Kang, Dong-Won

Karantoni, F. V.

Kargar, Fariborz

Kärger, Luise

Katunin, Andrzej

Khalili, Pooria

Khosravani, Mohammad Reza

Kim, Han Joo

Kim, Minkook

Kim, Soo Young

Kitada, Atsushi

Knite, Māris

Kołtunowicz, Tomasz Norbert

Korniejenko, Kinga

Korzeniewska, Ewa

Koutahzadeh, Negin

Koutavarapu, Ravindranadh

Koyanagi, Jun

Krauklis, Andrey E.

Kravchenko, Sergii

Kukla, Christian

Kulinich, Sergei

Kumanek, Bogumiła

Kumar, Dr. Santosh

Kumar, Vivek

Kuzmanovic, Maja

Kwon, Jae Sung

La, Duc Duong

Lamberti, Luciano

Łapińska, Barbara

Latko-Durałek, Paulina

Lavagna, Luca

Lee, Jaegeun

Lee, Min Wook

Leese, Hannah

Łękawa-Raus, Agnieszka

Lemu, Hirpa G.

Lenart, Małgorzata

Leonardi, Francesca

$\mathrm{Li}, \mathrm{He}$

Li, Jiaqi

Li, Lengwan

LI, Tianyi
Li, Ying

Li, Zheling

Liang, Tian

Lin, Guan

Lin, Tz-Feng

Lin, Zhibin

Liu, Dianzi

Liu, Qiang

Liu, Ting-Yu

Longo, Angela

López-Cela, Juan José

Majewska, Katarzyna

Maqbool, Muhammad

Marco, Carlotti

Marin, Elia

Marquez, Francisco M

Marra, Fabrizio

Marto, Carlos Miguel

Masato, Davide

Matykiewicz, Danuta

Mayyahi, Ahmed Al

McCoy, Thomas M.

Medved, Sergej

Mehrmashhadi, Javad

Meng, Fanbin

Merodio, Jose

Micó-Vicent, Bàrbara

Min, Rui

Miniaci, Marco

Mishra, Pawan Kumar

Mizera, Ales

Mohammed, Alyaa

Monti, Marco

Mortazavi, Bohayra

Moseenkov, Sergey I.

Mouritz, Adrian P

Moyseowicz, Adam

Mujtaba, Muhammad

Mukhin, Nikolay

Mun, Sung Cik

Musioł, Krzysztof

Muthuraj, Rajendran

Nabil, Bouazizi

Nair, Sandeep Sudhakaran

Nakashima, Naotoshi

Nakayama, Yuushou

Navarro, Elena

Neto, Maria

Ngo, Tri-Dung

Nguyen, Hoang

Nguyen, Ngoc A. 
$\mathrm{Ni}$, Xinchen

Niemczewska-Wojcik, Magdalena

NISHIKAWA, Masaaki

Norbert, Randl

Novak, Petr

Nowaczyk, Jacek

Olewnik-Kruszkowska, Ewa

Ombres, Luciano

Onuaguluchi, Obinna

Osswald, Tim

Ostapiuk, Monika

Pacelli, Settimio

Palanisamy, Selvakumar

Paleo Vieito, António Jose

Pall, Emoke

Panettieri, Enrico

Pantano, Maria

Papalou, Angeliki

Papanikos, Paraskevas

Paredes, Alejandro Javier

Paredes-Madrid, Leonel

Parisi, Filippo

Parmar, Kaushik

Pasquali, Michele

Pathak, Abhishek

Paul, Bere

Pawlak, Andrzej

Peeters, Daniël

Pegoretti, Alessandro

Pennetta, Antonio

Petrucci, Roberto

Pichandi, Subramani

Pierini, Filippo

Pinto, Gustavo

Plagge, Jan

Popescu, Vasilica

Pouranian, M. Reza

Price, Richard

Pruncu, Catalin I.

Ptiček Siročić, Anita

Puhan, Debashis

Puszka, Andrzej

Qu, Muchao

Quan, Gaofeng

Rackauskas, Simas

Radtke, Aleksandra

Rahman, Ashiqur

Rahman, Ashiqur X

Rahme, Kamil

Raimondo, Antonio

Ravi Nair, Jijeesh
Reddy, M.V. Venkatashamy

Refatul Islam, Mohammad

Renda, Simona

Rescalvo, Francisco J.

Rezaei, Shahed

Ribeiro, João

Rivera-Armenta, José Luis

Robert, Colin

Robles, Eduardo

Rocha, Mateus Garcia

Romanowicz, Paweł J.

Roscow, James

Rozylo, Patryk

Sagnelli, Domenico

Sahay, Rahul

Salazar, Yvette Allania

Saxena, Prateek

Sbardella, Francesca

Schabowicz, Krzysztof

Schintke, Silvia

Schmidt, Beata

Schneider, Matti

Seo, Young-Soo

Sepulveda, Abdon

Serpilli, Michele

Sgouros, Aristotelis

Shafiee, Ali

Shaha, Sugrib Kumar

Shakun, Alexandra

Sharma, Amit

Shevchenko, Vitaliy G.

Shibaev, Petr

Shin, Hyunseong

Shinde, Surendra Krushna

Sikder, Prabha

Singh, Manish

Skórczewska, Katarzyna

Skrifvars, Mikael

Ślosarczyk, Agnieszka

Smolka, Petr

Sommer, Drew

Song, Kenan

Sorrentino, Andrea

Spina, Roberto

Srinivasan, Gowrishankar

Staicu, Teodora

Staudinger, Ulrike

Strąkowska, Anna

Strzemiecka, Beata

Sun, Jinhua

Sun, Xiuxuan 
Sundaram, Rajyashree

Svetlana, Tretsiakova-McNally

Swiegers, Gerhard

Szostak, Marek

Taggart, David G.

Taglieri, Giuliana

Tamadon, Abbas

Tavares, Jason Robert

Teshnizi, Shokrollah Sharifi

Thapa, Mishal

Tian, Liang

Todaro, Francesco

Torres Marques, Antonio

Trakakis, George

Triantou, Marianna

Tripathi, Kumud Malika

Trzepiecinski, Tomasz

Tsirka, Kyriaki

Usman, Muhammad

Vannozzi, Lorenzo

Varona, Borja

Vekinis, George

Velu, Rajkumar

Vereshchaka, Alexey A.

Vinokurov, Sergey

Vivet, Alexandre

Vorndran, Elke

Voznyak, Yuri

Vryonis, Orestis

Vyazovkin, Sergey

Wang, Fuke

Wang, Jun

Wang, Lifeng

Wang, Po-Hsiang

Wang, Wei

Wang, Xiaohong

Wang, Yanshuai

Wei, Kongchang

Wei, Zhu

Weise, Joerg
Wen, Shipeng

Weston, David Peter

Wojnarowicz, Jacek

Wu, Kai

Wu, Liang

Wu, Zhen-Yu

Wudy, Katrin

Xi, Kai

Xiao, Xinxin

Xin, Haohui

$\mathrm{Xu}$, Hongyi

Yadav, Mithilesh

Yandouzi, Mohammed

Yaneva, Zvezdelina L.

Yang, Sen

Yang, Yang

Yazdani Sarvestani, Hamidreza

Yeboah, David

Yin, Kaiyang

You, Byoung He

Yu, Wenbin

Yuan, Chao

Zaharia, Catalin

Zekonyte, Jurgita

Zhang, Guoqiang

Zhang, Hai

Zhang, Meng

Zhang, Songlin

Zhang, Sunxiang

Zhang, Weizhao

Zhao, Yapu

Zheng, Qiye

Zholobko, Oksana

Zhou, Weiwei

Zhou, Wenbin

Zotti, Aldobenedetto

Zuev, Vjacheslav

Zukowski, Witold

Zygmuntowicz, Justyna 